\title{
State-of-the-art Forecasting Algorithms for Microgrids
}

\author{
Jie Ma, Xiandong Ma \\ Department of Engineering \\ Lancaster University \\ Lancaster. United Kingdom \\ j.ma6@lancaster.ac.uk
}

\begin{abstract}
As a controllable subsystem integrating with the utility, a microgrid system consists of distributed energy sources, power conversion circuits, storage units and adjustable loads. Distributed energy sources employ nonpolluted and sustainable resources such as wind and solar power in accordance with local terrain and climate to provide a reliable, consistent power supply for local customers. However, the electricity production in such a system is intermittent in nature, due to the time-varying weather conditions. Therefore, studies on accurate forecasting power generation and load demand are worthwhile in order to build a smart energy management system. The paper firstly reviews the forecasting algorithms for power supply side and load demand. The feasibly of the current control strategy is discussed. Finally, taking the wind turbine operational at Lancaster University campus as an example, results on power generation forecasting are presented by using a hybrid model combining Radial Basis Function and K-Means clustering. Development of new hybrid techniques aiming at improving model efficiency for online and real time forecasting will be one of the future research directions in this field.
\end{abstract}

Keywords- Microgrids; power generation forecasting; load demand forecasting; hybrid model; RBF-K-Means

\section{INTRODUCTION}

Conventional power station mainly depends on the non-renewable fossil fuels such as coal, oil and gas, which emits a large amount of greenhouse gas during the electricity production. The traditional, centralized electricity grid also causes losses in the transmission system. Moreover, the large-scale power outages cannot be avoided, when the mechanical or electrical faults happened to the over-centralized power station. It causes serious consequences on sensitive load in the utility.

The deployment of the distributed energy resource (DER) plays a significant role in strengthening a reliable and stable power line for the local end-user, reducing the production burden on power station and lowering the environment impacts. With more and more DER devices, energy storage devices and local loads integrating into the electricity grid, an innovative concept called 'microgrid' is proposed to group these equipment as a whole to connect the utility via the Point of Common Coupling(PCC) [1]. Microgrids enable to disconnect with the utility regarding themselves as an autonomous subsystem. However, there are still a lot of inevitable issues in microgrids. Wind and solar are uncontrollable and unstable resources, which is a big challenge for DER devices to provide a consistent supply for microgrids[2]. Similarly, the load consumption is affected by many factors, such as: weather and human behaviours. In order to balance the energy flow between the intermittent power generations and load demand under various situations, many intelligent approaches were developed to predict both sides more accurately.

This paper is organized as follow. Section 2 presents a detailed introduction of microgrid system structure and distribution system. The current methodologies used for short-term local power forecasting are reviewed in Section 3. Section 4 generalizes approaches on solving short-term load forecasting problem. In Section 5, a hybrid model integrating Radial Basis Function (RBF) with K-Means algorithm is presented to predict power in a more efficient way before conclusions are drawn.

\section{MICROGRIDS SYSTEM}

Building a modern, localized, small-scale grid in a limited geographical area maximizes the local resources and reduces the economy and energy losses of the power transmission. In terms of the utility, the development of microgrids is beneficial for enhancing local main grid stability, shifting the peak load demand, providing better voltage support, thus leading to an innovative low carbon technology.

\section{A. Microgrids structure \& operation modes}

A microgrid system is a cluster of microsources, storage devices and controllable AC and DC loads, providing heat and electricity for local users. A microgrid system typically comprises five components: DER sources, distribution systems (AC or DC bus), AC and DC loads, storage units and control and communication modules. The microsources typically rely on the distributed energy resources (DER) or/and conventional power generators, such as diesel generators. The DER devices include solar photovoltaic panels, wind turbines, combined heat and power (CHP), small hydro, biogas and fuel cells. On-site power generation aims to maximize benefits of local resource, decrease the power loss and economic loss due to the long-distance high-voltage transmission. The storage system in microgrids not only stores the excess power generated by the DERs, but also works as a stand-by power source to provide consistent power for the sensitive loads during the duration of operation mode switching. Furthermore, it ensures the balance between the power production and regular load demand. The commonly accepted storage devices involve

This work is funded by Lancaster University Centre for Global Eco-Innovation with the partnership of Microgrid Entrust LLP 
flywheels, batteries and super-capacitors. The control and communication modules consist of a central controller and power electronic interfaces (PEIs), used for realizing plug-and-play functionality to convert the power from DER devices to the bus voltages. The operation point of PEI is controlled by a central controller, as a master energy manager, which is responsible for automatic state switching, energy flow management and state monitoring of physical devices[3]

A microgrid system can operate in two modes, namely the grid-connected mode and the island mode. Under the grid-connected mode, the microgrid system makes benefits from selling the electricity to the utility, if the on-site power generation totally satisfies the end user need. Otherwise, it is required to purchase the electricity from the main grid in order to maintain its operation. If the power quality from the main grid is lower than an acceptable level, the microgrids should be seamlessly switched to island mode, for the sake of maintaining stability and avoiding the disturbance from the utility faults.

\section{B. Distribution systems}

The distribution system is referred to as a common bus to interconnect all physical devices in microgrids. Microgrids interconnect the main grid at the PCC, which is located on the primary side of the transformer. Generally, the operation mode in AC-microgrids follows the standard in the conventional AC grid. Fig 1 shows the microgrid architecture with AC-line configuration, where all of non-AC micro-generators and DC loads are converted to $50 \mathrm{~Hz}$ AC grid with power converter. However, considerable power loss during the power conversion and harmonic voltage are inevitable. A high frequency AC-link microgrids is proposed in [4], where a UPQC (Unified Power Quality Condition) is applied to achieve harmonic-free voltage and compensate for reactive power. The existing microgrid testbeds are mainly implemented on the AC grid, such as: CERTS testbed in America and MICROGRIDS project in Europe. CERTS testbed is a leading practical project launched by American Electrical Power, aiming at implementing the seamless transition between grid-connected and island operation in terms of reconnected and resynchronized process, maintaining the stability of voltage and frequency in microgrids on island condition. The testbed consists of three feeders for sensitive loads and a feeder for non-sensitive loads. In each sensitive feeder, subcontroller, breaker and more than one microsources are set to ensure a consistent power supply for sensitive loads Both sub-controller and central controller are introduced to realize 'plug and play' and 'peer-to-peer' functionality, respectively[5].

Meanwhile, DC-microgrid system has drawn a larger number of researcher's attentions in recent years. Many household devices and battery bank consume DC current, driving the development of DC-link microgrids. In conventional AC grid system, the power output of DCbased DER is transmitted to DC load through DC-ACDC power conversion, which causes considerable power losses. The power loss and financial cost are reduced substantially in DC-based microgrids due to no need of

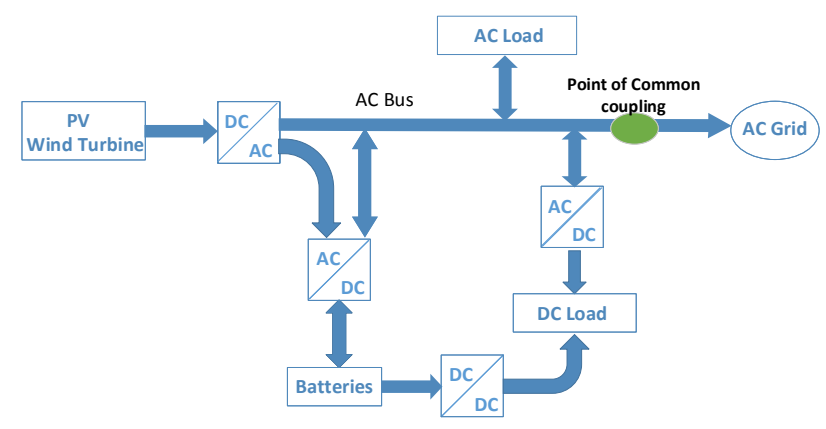

Figure 1 Vision of Microgrid in AC-link configuration

reactive power compensator and $\mathrm{DC} / \mathrm{AC}$ inverter.

In microgrids, the objective of energy management system is to employ intelligent control, state monitoring and device communication technology to construct a bidirectional energy flow system to properly dispatch the power production and user consumption. This has been made based on the forecasting results on the power side and load side. The forecasting technology is highly important to be developed, in order to set a proper energy management scheme.

\section{POWER GENERATION FORECASTING}

Wind turbines and photovoltaic panels are typical microsources in the microgrids, which have been feasibly installed. The wind and solar energy are weather driven resources, where their variability is ranging from minutely/hourly to yearly. The instability of renewable sources causes voltage fluctuation and intermittent power generation, posing the obstacle for maintaining power system operations and planning power system operations. Similarly, the electricity consumption is varied from season to season in a calendar year. Thus, developing appropriate forecasting technologies to predict power generation and user consumption is highly significant in order to address the supply and demand balance challenges. The forecasting time horizon is classified into the very short-term (from second to half an hour), shortterm (half an hour to 6 hours), medium-term (6 hour-1 day), long-term ( 1 day to 1 week), based on the power system management requirement. Very short-term forecasting aims at achieving dynamic control for microsources and load tracking. Short-term forecasting is used for scheduling energy flow among power source, load and storage devices. Medium-term and long-term forecasting are responsible for price settlement, load dispatch and maintenance scheduling, respectively.

A number of methodologies have been developed and combined to predict more precisely wind speed or/and wind power on different forecast horizons. The forecasting tools developed from many successful case studies are classified into three mainstream approaches: physical model, statistics model and computational intelligent model. The numerical weather prediction (NWP) model is the basis of physical approaches, where the variability of meteorological processes is described by atmospheric mesoscale model or global databases of meteor measurements. As for the statistics methods, the forecasting value has a linear correlation with historical data in specified time duration. The frequently used 
statistic methods include autoregression (AR), moving average (MA), autoregression moving average (ARMA), autoregression integrated moving average (ARIMA). Box-Jenkins approach is an effective tool to identify the components and parameters in time series while Kalman filter technique, also a parametric model, is implemented based on historical data. Hence, the intelligent approach considers the relationship from the input to the output as a 'black box', which consists of single model and hybrid model. The widely used single models include fuzzy logic, artificial neural network (ANN), support vector regression (SVR), wavelet transform (WT), genetic algorithm and expert system. The hybrid system is to integrate one or more algorithms together to pursue a higher forecasting accuracy. The most widely accepted hybrid model is adaptive neural fuzzy inference system (ANFIS).

\section{A. Statistic approaches}

With regard to the statistics approach, Kavasseri et al [6] utilized the fractional-ARIMA to improve forecasting accuracy on one-day and two-day horizon. The proposed statistic approach improved $3.08 \%$ and $6.52 \%$ regarding the square of Forecast Mean Square Error (FMSE), respectively. Ishikawa et al [7] applied Kalman Filter technology to estimate the parameters for wind speed and wind power forecasting. The wind speed forecasting model was developed by improving the NWP wind speed with Just in Time model. The initial predicted power output was obtained by substituting the forecasting wind speed into the wind turbine operation characteristics, while the final predicted wind power was optimized with Just In Time model. The simulation results showed that the maximum error rate for proposed model was lowest compared with single JIT model and other complex models.

\section{B. Intelligent algorithms}

In recent 20 years, most researchers have focused on computational algorithms in developing a non-linear map without considering the inner physical model. Many case studies indicate ANN is an effective tool to simplify the forecasting issue [8]-[13]. The biggest challenge for ANN application in wind power prediction is the selection of input variables. For example, in [10], after analysing weather and power generation in Central and South West Services Fort Davis wind farm, the author found wind speed, wind direction and individual characteristic of the wind turbine have a significant influence on the power output. The evaluation index identified the ANN model obviously outperforms the traditional models. Similarly, Mabel et al [8] considered different weather factors (humidity and wind speed) and turbine real operational hours as input variables. The proposed algorithm was practically used for the wind farm in India. Additionally, the accuracy of test model was varied from different structure of $\mathrm{ANN}$, as stated in [11], including the number of hidden layers, the number of neurones, iteration times and training algorithm.

\section{Hybrid models}

Cadenas et al [14] introduced a ARIMA-ANN hybrid model, where ARIMA model was used to obtain a roughly prediction value. Then, ANN was implemented to deal with the nonlinear tendency of forecasting error. The statistic outcomes showed the average mean absolute error (MAE) of the hybrid model is 0.2529 in each test site, which outperformans individual ANN (2.8131) and ARIMA (2.1451). In [15], Catalao et al combined the wavelet transform and ANFIS technology. The wavelet transform was utilized in the data pre-processing stage, where the trend and high-frequency information in original series were filtered into a group of constitutive power series. On the second stage, ANFIS was implemented for each series to estimate a set of parameters in membership function and consequent parameters under the fuzzy rule. Similarly, in [16], the author developed an evolutionary particle swarm optimization (EPSO) technology to adjust the parameters of membership function in ANFIS model. The average MAPE index showed that the proposed approach is $5.03 \%$, which was highly improved than other six traditional methods, involving persistence, ARIMA, NN, NNWT, NF, HPA, with $73.6 \%, 51.4 \%, 30.7 \%, 27.8 \%$, $24.2 \%, 7.0 \%$, respectively.

\section{Applications to solar power}

There are two mainstream approaches to estimate solar power generation. It is straightforward to establish a nonlinear map between the solar irradiance and solar power. Another method is to predict solar irradiance and calculate the corresponding power generation based on the characteristic of solar photovoltaic. Correspondingly, the intelligent algorithms have also been available for solar irradiance prediction[17]-[21]. Wang et al [17] considered integrating ANN into statistical feature parameters (ANN-SFP) to predict forecasting accuracy in uncertain weather conditions. The MAPE of ANN-SFP model on a cloudy day was $26.70 \%$, which is significantly improved than conventional ANN model $(81.11 \%)$. In [18], the author estimated the irradiance by calculating clearness index. The Radial Basis Function (RBF) and feedforward neural network model were investigated based on historical data in Omen. Geographic components (latitude, longitude, altitude) of the forecasting site, weather details (sunshine ratio) and calendar type (month of the year) were considered as influencing factors to clearness index. Chen et al. [21] proposed a hybrid model combining fuzzy logic and neural network, where temperature, sky information and solar irradiance level are grouped under the fuzzilization process. The accuracy rate of the hybrid model was considerably improved, compared with the traditional intelligent algorithms (fuzzy algorithm, neural network).

\section{SHORT-TERM LOAD FORECAST}

Short-term local load forecasting aims at planning an optimal electricity distribution schedule to satisfy the load consumption. Meanwhile, the load consumption is influenced by many factors, such as calendar type, weather, climate and special activity. Load forecasting techniques can be categorized as parametric methods, non-parametric methods and hybrid models, which are similar to power forecasting approaches.

\section{A. Statistic approaches}


Hong et al.[22]concentrated on a statistics approach to solve a time-varying nonlinear problem. The author classified independent input variables as quantitative variables, dummy variables and interactions. A trial-anderror approach was applied to determine the structure of a multiple linear regression model. The diagnostic statistics was employed to evaluate the performance of the model. Al-Hamadi et al.[23]presented a winter forecasting model on an hourly horizon based on state space and Kalman Filter. A state space model based on the previous hourly load value, temperature and wind parameters within a moving window was established. Afterwards, basic Kalman filter technique was applied to implement parameter identification. Then, other exogenous input components such as the calendar type were taken into account in order to improve the accuracy of the model.

\section{B. Intelligent algorithms}

Load forecasting issue can be treated as a black box problem, where its forecasting accuracy mostly depends on the size and accuracy of the dataset. In [24], authors reviewed a large number of papers about ANN applications in short term load forecasting and concluded that the best combination of input set involves the previously related load data, weather variables (dry bulb, dew point) and calendar types to predict load demand in a particular hour in a special day. Luis et al.[25]performed an ANN-based model into microgrids to forecast the hourly load in a day. The author only employed hourly load data of the previous day, the day type of a week, the month and total load of the forecasted day. Additionally, the Principal Component Analysis was implemented for outlier detection. In[26], Jaime et al. focused on their work in integrating the neural network with a classic control theory. On the first stage, the training process was implemented in an open-loop using historical load data, to determine the node weighting. At the second stage, the loop was closed to generate the one-hour ahead forecasted load by referring its current output load forecast and weather variables. Mamlook et al. [27] proposed a fuzzy inference model to express its outperformance, by taking into account the weather information. The fuzzy rules were designed from the local historical load data for a few years. Chenthur et al. [28] presented a fuzzy logic model, investigating the impacts of time, temperature, similar previous day load on the hourly forecasted error margin. The fuzzy rule base was designed using Mamdani implication. Khosravi et al. [23], [29]constructed a short term load forecasting model with the aims of decreasing load uncertainty and optimizing the power dispatch scheme.

The fuzzy logic applied in load forecasting issue has been proven to have its great advantages because it can appropriately describe ambiguous input variables, such as weather parameters and calendar type. However, it requires collecting a large amount of historical load data, weather data in order to obtain the fuzzy rules. Furthermore, the fuzzy rule is normally obtained from the specific area, which might be infeasible in a large scale area.

\section{Hybrid models}

Firas et al. [30]presented a fuzzy-logic-related hybrid model consisting of two stages. At the first stage, Interval Type 2 Fuzzy Logic System (IT2 FLS), as an extension of the ordinary fuzzy logic concept, was applied to handle the uncertainty in forecasting accuracy or unexpected exogenous outputs. The independent forecasted models for summer and winter were developed respectively. At the second stage, a genetic algorithm was used for optimizing parameters in IT2 FLS model, in order to achieve the satisfactory stopping criteria. Cevic et al.[31]developed ANFIS to predict hourly load consumption. In this research, the data set of the input variables considered the impact of historical load, weather and season factors on the forecasted mode. Hence, the data sets were grouped with various week time and off-week time. It was observed that the MAPE of the hybrid model was up to $1.7 \%$, which demonstrates its outperformance than a single fuzzy logic.

\section{HYBRID MODEL FOR POWER PREDICTION}

Among the existing approaches reviewed in this paper, technologies based on the nonlinear hybrid model have been widely accepted. Here, the work about power generation forecasting was carried out to prepare for the future overall microgrid system implementation. As an example, a commonly accepted algorithm-Radial Basis Function (RBF) is employed. This algorithm itself possesses an ANN structure with single hidden layer, which has fast learning capability as the output weightings are determined by least square normal equation, without iterative process being utilized.

\section{A. Radial Basis Function-K-Means model}

Radial Basis Function (RBF) network, as a feedforward neural network, consists of an input layer, one hidden layer and an output layer. The input neuron feed the value to each hidden neurons directly. An RBF network positions a variable number of hidden neurons, optimized by training process. The activation function for neurons in hidden layer is described by RBF whose output is inversely proportional to the distance with the centre of the neurons. The widely accepted RBF is Gaussian function, with centred on a point as many varied dimensional radials as the number of input variables. The centre and width are determined during the training process with K-Means clustering algorithm. The hidden neuron computes the Euclidean distance of the test case from the neuron's centre and then calculates the resulting value based on RBF function using this distance and width value, which is denoted as

$$
\varphi_{i}(r)=\exp \left(-\frac{r^{2}}{2 \sigma^{2}}\right)
$$

where $\varphi_{i}$ is the output value of the $i$-th hidden neuron, $r$ is the Euclidean distance from neuron's centre to sample data, $\sigma$ is the width of Gaussian function corresponding to the input variable. The best predicted value for the new point is found by summing the output value of the hidden neuron multiplied by weights computed for each neuron, which is described as:

$$
H(x)=\sum_{i=1}^{M} w_{i} \varphi_{i}(r)+b_{0}
$$


where $H$ is the predicted value of RBF network, $M$ is the number of hidden layer, $w_{i}$ is the weighting coefficients between the $i$-th hidden neuron and output layer, $b_{0}$ is the bias term at output node. Least square approach is utilized to figure out weighting coefficient upon the given output dataset.

K-Means clustering is used for relocating the centre of Gaussian function with neighbourhood rule, when a new sample coming for training or testing. The initial centroids are selected randomly from $k$ different data points, where $k$ is the number of hidden layer neurons. The Euclidean distance between the initial centroid and rest data points in the dataset is calculated for sample classification. Afterwards, the updated centroids are relocated with the current clustering results. The new classification with updated centroid is implemented iteratively to update clustering results; when centroids for different clusters do not move any more, the final clustering results are figured out.

\section{B. Case study}

The historical wind turbine dataset at Lancaster University campus is employed to identify the feasibility and efficiency of proposed hybrid approach. The historical wind speed and power output data from $22^{\text {nd }}$ Nov 2012 to $22^{\text {nd }}$ Jan 2013 with the resolution of 10 minutes are employed for training the RBF network. The data ranging from $23^{\text {rd }}$ Jan 2013 to $23^{\text {rd }}$ Feb 2013 is introduced to evaluate the performance of the trained RBF network. A novel evaluation index so-called Normalized Root Mean Square Error (NRMSE) is introduced to express the error between the real output and output from proposed forecasting model, which is denoted as

$$
N R M S E=\sqrt{\sum_{i=1}^{P}\left(y_{i}-f\left(x_{i}\right)\right)^{2} / \sum_{i=1}^{P}\left(y_{i}-\bar{y}\right)^{2}}
$$

where $\bar{y}$ is the mean value of real output $y_{i}, P$ is the total data number. $f\left(x_{i}\right)$ denotes predicted value[32]. The coefficient of determination is deployed here to evaluate how well a model explain the actual output data and is defined as

$$
R^{2}=1-\frac{S S_{\text {res }}}{S S_{\text {total }}}
$$

where $S S_{\text {res }}$ is the sum of squared predicted output residuals, $S S_{\text {total }}$ is the total sum of squares. If $R^{2}$ tends to unity, it indicates the model provide a good fit to real case. However, when $R^{2}$ close to zero, it indicates the theoretic model fail to fit the real model. [33]

The hybrid model integrating $\mathrm{RBF}$ and $\mathrm{K}$-means clustering is implemented within following steps

i) Data pre-processing to eliminate those obvious abnormal data, faulty data to improve the training data accuracy.

ii) Initial centroids selection and centroids relocation for Gaussian function with K-means algorithm.

iii) $\mathrm{RBF}$ network determination with least square method during the training process.

iv) Model evaluation.

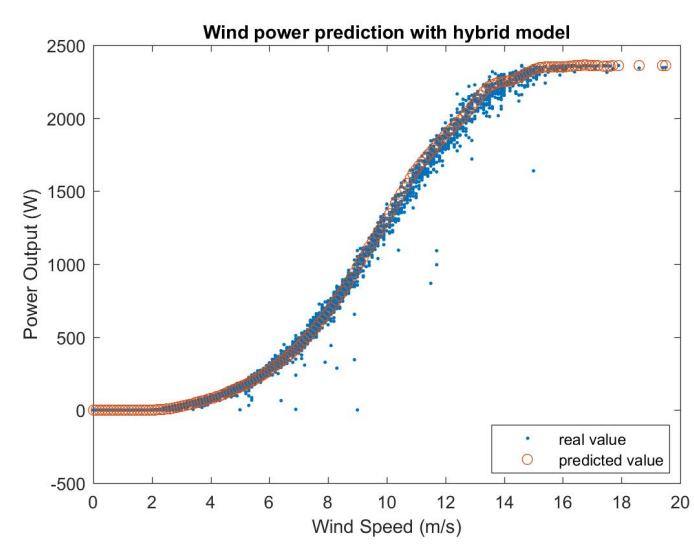

Figure 2 Wind power prediction with the RBF hybrid model

The simulation is implemented in the Intel Core i7 CPU with frequency of $2.59 \mathrm{GHz}$. Fig 2 describes the test results of RBF neural network compared with real power output. It can be seen that the hybrid model well fit the nonlinear S-curve showing how the power output from the turbine varies with steady wind speed. Mathematically, its coefficient of determination is 0.9955 , which outperforms conventional ANN network 0.9934. The NRMSE of the hybrid mode is 0.0741. More importantly, RBF network with single hidden layer considerably reduce by 10 times in running time compared with the conventional ANN Feedforward (FF) network, based on the same datasets. The running time comparison is given in Table 1 . The results $d$ the high efficiency of the proposed hybrid approach.

TABLE I Running time of hybrid model and FF

\begin{tabular}{|c|c|c|}
\hline Running Time(second) & Training & Testing \\
\hline RBR-K-means network & 0.8085 & 0.10112 \\
\hline Feedforward neural network & 9.2274 & 1.1166 \\
\hline
\end{tabular}

\section{CONCLUSION}

This paper reviews various forecasting methods from the power supply to load demand for microgrids. Microgrids technologies are reviewed, involving its key components, operation modes and distribution buses. Short-term forecasting methodologies for power generation and load have been developed to construct an intelligent microgrid to balance power supply and load. Most of methodologies are classified into statistics approaches, computing algorithms and hybrid models combining two or more algorithms. The most influenced factors for power generation forecasting are associated with weather information, including wind speed and solar irradiance. The load consumption is affected by weather condition, calendar type and human behaviours. A hybrid model combining RBF network and K-Means clustering is employed to improve the training efficiency compared with conventional ANN method. The preliminary results are promising.

\section{ACKNOWLEDGEMENT}

The authors are grateful to Lancaster University's Facility office for providing campus wind turbine operation data and Lancaster Environment Centre for providing weather data. This work is funded by Lancaster 
University Centre for Global Eco-Innovation with the partnership of Microgrid Entrust LLP.

\section{REFERENCES}

[1] U.S. Department of Energy, "DOE Microgrid Workshop Report," 2011.

[2] X. Ma, Y. Wang, and J. Qin, "Generic model of a community-based microgrid integrating wind turbines, photovoltaics and CHP generations," Appl. Energy, vol. 112, pp. 1475-1482, 2013.

[3] L. Mariam, M. Basu, and M. F. Conlon, "Microgrid: Architecture, policy and future trends," Renew. Sustain. Energy Rev., vol. 64, pp. 477-489, 2016.

[4] H. J. Su, G. W. Chang, S. Ranade, and H. J. Lu, "Modeling and simulation of an ac microgrid for harmonics study," in IEEE International Conference on Harmonics and Quality of Power, 2012, pp. 668-672.

[5] R. H. Lasseter et al., "CERTS microgrid laboratory test bed," IEEE Trans. Power Deliv., vol. 26, no. 1, pp. 325332, 2011.

[6] R. G. Kavasseri and K. Seetharaman, "Day-ahead wind speed forecasting using f-ARIMA models," Renew. Energy, vol. 34, no. 5, pp. 1388-1393, 2009.

[7] T. Ishikawa and T. Namerikawa, "Short-Term Wind Power Prediction for Wind Turbine via Kalman Filter based on JIT Modeling," in SICE Annual Conference, 2013.

[8] M. Carolin Mabel and E. Fernandez, "Analysis of wind power generation and prediction using ANN: A case study," Renew. Energy, vol. 33, no. 5, pp. 986-992, 2008.

[9] P. Flores, A. Tapia, and G. Tapia, "Application of a control algorithm for wind speed prediction and active power generation," Renew. Energy, vol. 30, no. 4, pp. 523-536, 2005.

[10] L. Shuhui, W. Donald C, O. Edgar A, and Michael G. Giesselmann, "Using Neural Networks to EstimateWind Turbine Power Generation," IEEE Trans. ENERGY Convers., vol. 16, no. 3, pp. 276-282, 2001.

[11] T. Kaur, S. Kumar, and R. Segal, "Application of Artificial Neural Network for Short Term Wind Speed Forecasting," in Biennial International Conference on Power and Energy Systems, 2016.

[12] M. A. Mohandes and S. Rehman, "A neural networks approach for wind speed prediction," Renew. Energy, vol. 13 , no. 3, pp. 345-354, 1998.

[13] M. C. Alexiadis, P. S. Dokopoulos, H. S. Sahsamanoglou, and I. M. Manousaridis, "Short-term forecasting of wind speed and related electrical power," Sol. Energy, vol. 63, no. 1, pp. 61-68, 1998.

[14] E. Cadenas and W. Rivera, "Wind speed forecasting in three different regions of Mexico, using a hybrid ARIMAANN model," Renew. Energy, vol. 35, no. 12, pp. 2732 $2738,2010$.

[15] J. P. S. Catalão, H. M. I. Pousinho, and V. M. F. Mendes, "Hybrid intelligent approach for short-term wind power forecasting in Portugal," IET Renew. Power Gener., vol. 5, no. 3, p. 251, 2011.

[16] J. P. S. Catalao, G. J. Osorio, and H. M. I. Pousinho, "Short-term wind power forecasting using a hybrid evolutionary intelligent approach," in Intelligent System Application to Power Systems (ISAP), 2011 16th International Conference on, 2011, pp. 1-5.

[17] F. Wang, Z. Mi, S. Su, and H. Zhao, "Short-term solar irradiance forecasting model based on artificial neural network using statistical feature parameters," Energies, vol. 5, no. 5, pp. 1355-1370, 2012.

[18] A. S. S. Dorvlo, J. A. Jervase, and A. Al-Lawati, "Solar radiation estimation using artificial neural networks," Appl. Energy, vol. 71, no. 4, pp. 307-319, 2002.

[19] A. Mellit and A. M. Pavan, "A 24-h forecast of solar irradiance using artificial neural network: Application for performance prediction of a grid-connected PV plant at
Trieste, Italy," Sol. Energy, vol. 84, no. 5, pp. 807-821, 2010.

[20] F. Grimaccia, S. Leva, M. Mussetta, and E. Ogliari, "Analysis and Validation of ANN PV Power Output Forecasting At 24 Hours Ahead," in ElectrIMACS, 2014.

[21] S. X. Chen, H. B. Gooi, and M. Q. Wang, "Solar radiation forecast based on fuzzy logic and neural networks," Renew. Energy, vol. 60, pp. 195-201, 2013.

[22] T. Hong, M. Gui, M. Baran, "Modeling and Forecasting Hourly Electric Load by Multiple Linear Regression with Interactions," in Power and Energy Society General Meeting, 2010, pp. 1-8.

[23] H. M. Al-Hamadi and S. A. Soliman, "Short-term electric load forecasting based on Kalman filtering algorithm with moving window weather and load model," Electr. Power Syst. Res., vol. 68, no. 1, pp. 47-59, 2004.

[24] M. Q. Raza and A. Khosravi, "A review on artificial intelligence based load demand forecasting techniques for smart grid and buildings," Renew. Sustain. Energy Rev., vol. 50, pp. 1352-1372, 2015.

[25] L. Hernandez, C. Baladrón, J. Aguiar, B. Carro, A. Sanchez-Esguevillas, and J. Lloret, "Short-Term Load Forecasting for Microgrids Based on Artificial Neural Networks," Energies, vol. 6, no. 3, pp. 1385-1408, 2013.

[26] J. Buitrago and S. Asfour, "Short-Term Forecasting of Electric Loads Using Nonlinear Autoregressive Artificial Neural Networks with Exogenous Vector Inputs," Energy, vol. 10, no. 40, pp. 1-24, 2017.

[27] R. Mamlook, O. Badran, and E. Abdulhadi, "A fuzzy inference model for short-term load forecasting," Energy Policy, vol. 37, no. 4, pp. 1239-1248, 2009.

[28] S. Chenthur Pandian, K. Duraiswamy, C. Christober Asir Rajan, and N. Kanagaraj, "Fuzzy approach for short term load forecasting," Electr. Power Syst. Res., vol. 76, no. 6, pp. 541-548, 2006.

[29] A. Khosravi, S. Nahavandi, and D. Creighton, "Short term load forecasting using Interval Type-2 Fuzzy Logic Systems," in IEEE International Conference on Fuzzy Systems (FUZZ-IEEE 2011), 2011, pp. 502-508.

[30] F. M. Tuaimah and H. M. A. Abbas, "Iraqi Short Term Electrical Load Forecasting Based On Interval Type-2 Fuzzy Logic," Int. J. Electr. Comput. Energ. Electron. Commun. Eng., vol. 8, no. 8, pp. 1255-1261, 2014.

[31] H. Huseyin Cevic and M. Cunkas, "Short-term load forecasting using fuzzy logic and ANFIS," Neural Comput Appl., vol. 26, no. 6, pp. 1355-1367, 2015.

[32] M. Awad, H. Pomares, I. Rojas, O. Salameh, and M. Hamdon, "Prediction of Time Series Using RBF Neural Networks: A New Approach of Clustering.," Int. Arab J. Informationa Technol., vol. 6, no. 2, pp. 138-144, 2009.

[33] P. Cross and X. Ma, "Model-based and fuzzy logic approaches to condition monitoring of operational wind turbines," Int. J. Autom. Comput., vol. 12, no. 1, pp. 25-34, 2015. 